\title{
Enzyme-responsive doxorubicin release from dendrimer nanoparticles for anticancer drug delivery
}

This article was published in the following Dove Press journal:

International Journal of Nanomedicine

28 August 2015

Number of times this article has been viewed

\author{
Sang Joon Lee ${ }^{1, *}$ \\ Young-II Jeong ${ }^{2, *}$ \\ Hyung-Kyu Park ${ }^{3}$ \\ Dae Hwan Kang 2,4 \\ Jong-Suk $\mathrm{Oh}^{3}$ \\ Sam-Gyu Lee ${ }^{5}$ \\ Hyun Chul Lee ${ }^{3}$
}

'Department of Biomedical Sciences, Chonnam National University Medical School, Gwangju, ${ }^{2}$ Biomedical Research Institute, Pusan National University Hospital, Busan, ${ }^{3}$ Department of Microbiology, Chonnam National University Medical School, Gwangju, ${ }^{4}$ Research Institute for Convergence of Biomedical Science and Technology, Pusan National University Yangsan Hospital, Gyeongnam, ${ }^{5}$ Department of Physical and Rehabilitation Medicine, Chonnam National University Medical School, Gwangju, Republic of Korea

*These authors contributed equally to this work

Correspondence: Hyun Chul Lee Department of Microbiology, Chonnam National University Medical School, Gwangju 50I-746, Republic of Korea $\mathrm{Tel}+82622204133$

Fax +8262 2287294

Email hclee@chonnam.ac.kr

Dae Hwan Kang

Biomedical Research Institute, Pusan

National University Hospital, 179

Gudeok-ro, Seo-gu, Pusan 602-739,

Republic of Korea

$\mathrm{Tel}+82553603870$

Fax +82 553603879

Email sulsulpul@naver.com
Background: Since cancer cells are normally over-expressed cathepsin B, we synthesized dendrimer-methoxy poly(ethylene glycol) (MPEG)-doxorubicin (DOX) conjugates using a cathepsin B-cleavable peptide for anticancer drug targeting.

Methods: Gly-Phe-Leu-Gly peptide was conjugated with the carboxylic acid end groups of a dendrimer, which was then conjugated with MPEG amine and doxorubicin by aid of carbodiimide chemistry (abbreviated as DendGDP). Dendrimer-MPEG-DOX conjugates without Gly-PheLeu-Gly peptide linkage was also synthesized for comparison (DendDP). Nanoparticles were then prepared using a dialysis procedure.

Results: The synthesized DendGDP was confirmed with ${ }^{1} \mathrm{H}$ nuclear magnetic resonance spectroscopy. The DendDP and DendGDP nanoparticles had a small particle size of less than $200 \mathrm{~nm}$ and had a spherical morphology. DendGDP had cathepsin B-sensitive drug release properties while DendDP did not show cathepsin B sensitivity. Further, DendGDP had improved anticancer activity when compared with doxorubicin or DendDP in an in vivo CT26 tumor xenograft model, ie, the volume of the CT26 tumor xenograft was significantly inhibited when compared with xenografts treated with doxorubicin or DendDP nanoparticles. The DendGDP nanoparticles were found to be relatively concentrated in the tumor tissue and revealed stronger fluorescence intensity than at other body sites while doxorubicin and DendDP nanoparticles showed strong fluorescence intensity in the various organs, indicating that DendGDP has cathepsin B sensitivity.

Conclusion: DendGDP is sensitive to cathepsin B in tumor cells and can be used as a cathepsin B-responsive drug targeting strategy. We suggest that DendGDP is a promising vehicle for cancer cell targeting.

Keywords: cathepsin B, CT26 cell, enzyme-sensitive dendrimer, tumor targeting, tetra peptide

\section{Introduction}

Drug targeting to a specific site of action is still a major problem in the field of biomedicine and drug delivery. ${ }^{1,2}$ Because most drugs diffuse freely throughout the body, conventional drug formulations induce a variety of side effects in the human body and lose their intrinsic therapeutic effects. The serious adverse effects of anticancer drugs in the clinical setting are often mentioned in the literature. ${ }^{3-5}$ Site-specific targeting of anticancer drugs to tumor tissues remain a major challenge in cancer chemotherapy. ${ }^{5-7}$ To overcome these drawbacks, nanomaterials, nanoparticles, polymer-drug conjugates, and nanodevices have been extensively investigated in the last 2 decades. $^{8-12}$ Nanomaterials and devices based on polymeric conjugates have been widely investigated 
in an effort to facilitate drug trafficking efficiently in tumor tissues. Enhanced tumor targeting by nanoparticles is based on the enhanced permeation and retention (EPR) effect in tumor tissues. ${ }^{10}$ Then, nanoparticles are selectively targeted at the tumor tissues through physicochemical and biological pathway. ${ }^{10}$ Compared with conventional drug carriers, nanomaterials such as nanotubes, polymeric micelles, dendrimers, liposomes, and nanoparticles have several advantages, including a large surface area, ease of chemical modification, a small diameter for site-specific targeting of anticancer drugs, solubilization of lipophilic drugs, and physicochemical functionality. ${ }^{8-16}$ For example, their large surface area and small particle size enable conjugation of cancer cell-targeting ligands, such as monoclonal antibodies, folic acid, and RGD peptides. ${ }^{15-18}$

Among the nanomaterials, dendrimers have been extensively investigated in the biomedical field. ${ }^{19-23}$ Dendrimers are a class of macromolecular hyperbranched polymers having an intrinsic structure composed of a core, branches, void spaces, and terminal groups. ${ }^{19}$ Due to their structural peculiarity, dendrimers have better physicochemical behavior than linear or branched polymers. ${ }^{21,22}$ For example, dendrimers tend to have lower viscosity, unique molecular behavior, and a lower hydrated radius when compared with linear polymers. ${ }^{21,22}$ Due to these peculiarity, dendrimer is able to manipulate at mild condition and it can be endowed with multifunctionality. ${ }^{19}$ In particular, dendrimers is are suitable vehicles for anticancer drugs and a targeting moiety because they have multivalent functional groups on their terminal groups. This characteristic enables dendrimers to conjugate with various molecules, such as anticancer drugs and poly(ethylene glycol) (PEG), and a targeting moiety and peptide can be simultaneously conjugated to the dendrimer. ${ }^{20,23,24}$ In a report by He et al an RGD peptide for targeting cancer cells, PEG for avoidance of the reticuloendothelial system, and an anticancer drug were simultaneously introduced to a poly(amidoamine) (PAMAM) dendrimer. ${ }^{24}$ These authors showed that a multifunctional dendrimer efficiently targeted U87MG cells via a receptor-mediated pathway and could inhibit cell viability. Further, Kaminskas et al reported that PEGylated dendrimer-methotrexate conjugates having matrix metalloproteinase-2/9 cleavable peptide are efficiently targeted to tumor cells, suppressed growth of cancer cells, and circulated in the blood stream for a longer period. ${ }^{25}$

In this study, we synthesized doxorubicin-conjugated dendrimer nanoparticles using glycylphenylalanyl-leucylglycine tetrapeptide (Gly-Phe-Leu-Gly, GFLG) for cathepsin B-responsive drug targeting of cancer cells. Cathepsin B, which is a lysosomal cysteine protease, is known to degrade extracellular matrix components during invasion and metastasis of cancer cells. ${ }^{26}$ To determine if this conjugate could be considered a promising vehicle for cancer-targeting drug carriers, we carried out a physicochemical/biological analysis in vitro and tested this conjugate in an animal tumor xenograft model using CT26 colorectal carcinoma cells.

\section{Materials and methods \\ Materials}

The dendrimer (NTN1956) was purchased from Frontier Scientific Inc (USA). GFLG tetrapeptide was purchased from Peptron Co (Korea). Methoxy PEG (MPEG) amine (molecular weight 2,000 g/mol) was obtained from Sunbio Chem Co (Korea). Doxorubicin $\mathrm{HCl}$ was purchased from LC Laboratories (USA). Cathepsin B (from bovine spleen), dimethyl sulfoxide (DMSO), triethylamine, 3-(4,5-dimethylthiazol2-yl)-2,5-diphenyl tetrazolium bromide (MTT), N-(3dimethylaminopropyl)-N-ethylcarbodiimide hydrochloride (EDAC), and N-hydroxysuccinimide (NHS) were sourced from Sigma Chemicals Co (USA). Spectra/Por ${ }^{\mathrm{TM}}$ dialysis membranes (molecular weight cut-off 2,000 and 8,000 $\mathrm{g} / \mathrm{mol}$ ) were purchased from Spectrum Laboratories Inc (USA). Roswell Park Memorial Institute (RPMI) 1640 medium and fetal serum bovine albumin were purchased from Gibco (Invitrogen Co, USA). All other chemicals used were high-performance liquid chromatography or extra pure grade.

\section{Synthesis of dendrimer nanoparticles Dendrimer-peptide conjugates}

First, $13.4 \mathrm{mg}$ of dendrimer was dissolved in DMSO with 12 equivalents of EDAC and NHS and magnetically stirred for 6 hours. Twelve equivalents of tetrapeptide were then added to this reaction and stirred for a further 48 hours. To remove the unreacted chemicals and byproducts, the reactants were introduced into a dialysis membrane (molecular weight cut-off 2,000 g/mol) and dialyzed against deionized water for 2 days, with exchange of water at 2-3-hour intervals. The dialyzed solution was then lyophilized for 3 days, after which a white powder (dendrimer-peptide conjugates) was obtained. The final yield of conjugates was approximately $91 \%(\mathrm{w} / \mathrm{w})$.

\section{Dendrimer-peptide-MPEG conjugates}

Sixty milligrams of the dendrimer-peptide conjugate in DMSO were mixed with 4 equivalents of EDAC/NHS and stirred for 6 hours. Eight milligrams of MPEG-amine was then added to this reaction and stirred for a further 48 hours. To remove the unreacted MPEG-amine, the reactants were introduced into a dialysis membrane (molecular weight 
cut-off $8,000 \mathrm{~g} / \mathrm{mol}$ ) and dialyzed against deionized water for 2 days, with exchange of water at 2-3-hour intervals. The dialyzed solution was lyophilized for 3 days, after which a white powder was obtained, with a product yield of approximately $93 \%(w / w)$.

\section{Dendrimer-peptide-MPEG-DOX conjugates (DendGDP)}

First, $140 \mathrm{mg}$ of dendrimer-peptide-MPEG in DMSO was mixed with 8 equivalents of EDAC/NHS and stirred for 3 hours. Eight equivalents of doxorubicin HCL with trace amounts of triethylamine were then added to this reaction, with magnetic stirring for 48 hours. The reactants were then dialyzed against water using a dialysis membrane (molecular weight cut-off $8,000 \mathrm{~g} / \mathrm{mol}$ ) and dialyzed against deionized water for a further 2 days, with exchange of water at 3 hour intervals.

\section{Dendrimer-MPEG-DOX conjugates (DendDP)}

First, $13.4 \mathrm{mg}$ of dendrimer in DMSO was mixed with 4 equivalents of EDAC/NHS and magnetically stirred for 6 hours. Eighty milligrams of MPEG-amine was then added to this reaction, with stirring for another 48 hours. To remove the unreacted MPEG-amine, the reactants were introduced into a dialysis membrane (molecular weight cutoff $8,000 \mathrm{~g} / \mathrm{mol}$ ) and dialyzed against deionized water for 2 days following lyophilization for 3 days (the product yield of dendrimer-MPEG conjugates was approximately $92 \%$ [w/w]). Next, $93 \mathrm{mg}$ of the dendrimer-MPEG conjugates in DMSO were mixed with 8 equivalents of doxorubicin HCL and trace amounts of triethylamine. This reaction was then magnetically stirred for 48 hours, followed by dialysis against deionized water for 2 days, and then solution was lyophilized for 3 days.

\section{Analysis}

${ }^{1} \mathrm{H}$ nuclear magnetic resonance (NMR) spectroscopy (using a $500 \mathrm{MHz}$ FT-NMR spectrometer, Varian Inc, USA) was used to analyze the dendrimer and its related conjugates.

\section{Preparation and characterization of nanoparticles}

Fifty milligrams of DendGDP or DendDP dissolved in $4 \mathrm{~mL}$ of DMSO was slowly dropped into $10 \mathrm{~mL}$ of deionized water. This solution was dialyzed (dialysis membrane molecular weight cut-off $8,000 \mathrm{~g} / \mathrm{mol}$ ) against water for 2 days to remove the organic solvent, with exchange of water at 2-3-hour intervals. The morphology of the nanoparticles was observed using a transmission electron microscope
(H-7600, Hitachi Instruments Ltd, Japan). The size of the dendrimer nanoparticles was analyzed using a Nano-ZS (Malvern Instruments, UK).

\section{Drug release study}

Five milligrams of the lyophilized nanoparticles were reconstituted in $1 \mathrm{~mL}$ of deionized water and then diluted with $4 \mathrm{~mL}$ of sodium acetate buffer ( $\mathrm{pH}$ 5.4) with or without cathe$\mathrm{psin} \mathrm{B}(50 \mathrm{U})$. The nanoparticle solution was introduced into a dialysis membrane (molecular weight cut-off 2,000 g/mol). The dialysis membrane was then introduced into a $50 \mathrm{~mL}$ Falcon tube with $40 \mathrm{~mL}$ of buffer solution. The drug release study was performed in a shaking incubator (at $100 \mathrm{rpm}$ and $37^{\circ} \mathrm{C}$ ). At predetermined time intervals, $1 \mathrm{ml}$ of media was taken to measure concentration of the released drug and then the whole media were replaced with fresh media to prevent drug saturation. The doxorubicin concentration released was analyzed using an UV-1801 spectrophotometer (Shimadzu Co, Japan) at $479 \mathrm{~nm}$.

\section{In vitro cell culture study}

For the in vitro cell culture study, CT26 mouse colorectal carcinoma cells were obtained from the Korean Cell Line Bank and maintained in RPMI 1640 medium supplemented with $10 \%(\mathrm{v} / \mathrm{v})$ fetal bovine serum and antibiotics in a $5 \%$ $\mathrm{CO}_{2}$ incubator at $37^{\circ} \mathrm{C}$.

Doxorubicin-resistant CT26 cells were prepared as reported previously. ${ }^{18}$ CT26 cells $\left(1 \times 10^{5}\right)$ in serum-free RPMI medium were exposed to doxorubicin for 3 hours, after which the medium was replaced with fresh RPMI 1640 medium supplemented with fetal bovine serum/antibiotics. Exposure to doxorubicin was repeated 2-3 times at 3-day intervals for 3 months, with the doxorubicin concentration gradually being increased from $0.001 \mu \mathrm{g} / \mathrm{mL}$ to $1.0 \mu \mathrm{g} / \mathrm{mL}$.

The in vitro cell viability study was performed as follows. CT26 cells $\left(3 \times 10^{4}\right)$ were seeded into 96 -well plates, cultured overnight, and then treated with doxorubicin, DendDP, or DenGDP. For the doxorubicin group, doxorubicin $\mathrm{HCl}$ in DMSO was diluted with RPMI 1640 medium (final DMSO concentration less than $0.5 \%$ ) and added to the 96-well plate containing the cultured CT26 cells. For the DendDP and DendGDP groups, the nanoparticles were reconstituted in deionized water and then diluted with RPMI 1640 medium for treatment of CT26 cells. The cells used for each treatment group were incubated for a further 24 hours in a $\mathrm{CO}_{2}$ incubator. MTT $(30 \mu \mathrm{L}, 5 \mathrm{mg} / \mathrm{mL}$ in PBS [phosphate-buffered saline]) was then added to the 96-well plates, with incubation for a further 4 hours. Finally, $0.1 \mathrm{~mL}$ 
of DMSO was added to dissolve the formazan crystals generated in viable cells. Cell viability was then evaluated by measurement of absorbance (560 nm test/630 nm reference) using a microplate reader (Molecular Devices Inc, USA).

\section{In vivo fluorescence imaging}

To study the biodistribution of the dendrimer nanoparticles, doxorubicin or nanoparticles were injected intravenously via the tail vein into each mouse at a dose of $10 \mathrm{mg} / \mathrm{kg}$ as a DOX concentration. Injection volume was $100 \mu \mathrm{l}$. One and 2 days later, the mice were observed using a Maestro ${ }^{\text {TM }} 2$ small animal imaging system (Cambridge Research and Instrumentation Inc, USA). The mice were sacrificed 48 hours after injection of the study formulations to observe the distribution of the nanoparticles in the organs.

\section{In vivo antitumor activity study}

The antitumor activity of doxorubicin and the nanoparticles was also evaluated an in vivo tumor xenograft model using male nude BALB/c mice (aged 4-5 weeks, weight $20 \mathrm{~g}$ ).

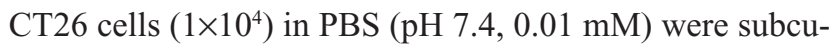
taneously injected in the dorsum of each mouse. Ten days later, the animals were divided into four groups, ie, control, doxorubicin, DendDP, and DendGDP, with each treatment group containing 4-5 mice. PBS (control), doxorubicin, or nanoparticles (containing a doxorubicin dose equivalent to $5 \mathrm{mg} / \mathrm{kg}$ ) were injected intravenously via the tail vein. The injection volume was $0.1 \mathrm{~mL}$. The day of the first injection was designated day $0 ; 3$ days later, a second intravenous dose of doxorubicin or nanoparticles was injected. Tumor growth was monitored at 3-5-day intervals using Vernier calipers. The tumor volume was calculated using the following equation: $\left(\right.$ length $\times$ width $\left.^{2}\right) / 2$. All animal experiments were carried out following the guidelines approved by the Committee for the Care and Use of Laboratory Animals at Chonnam National University.

\section{Statistical analysis}

All results are expressed as the mean \pm standard deviation. The statistical analysis was performed using the Student's

Table I Characterization of dendrimer nanoparticles

\begin{tabular}{lll}
\hline & $\begin{array}{l}\text { Drug content } \\
(\%, w / w)\end{array}$ & $\begin{array}{l}\text { Mean particle } \\
\text { size }(\mathbf{n m})\end{array}$ \\
\hline DendDP & 24.8 & 54 \\
DendGDP & 17.6 & 63 \\
\hline
\end{tabular}

Abbreviations: DendGDP, dendrimer-MPEG-DOX conjugate with GFLG peptide linkage; DendDP, dendrimer-MPEG-DOX conjugate; DOX, doxorubicin; MPEG, methoxy poly(ethylene glycol); GFLG, Gly-Phe-Leu-Gly. $t$-test and results were considered to be statistically significant when the $P$-value was less than 0.05 .

\section{Results}

\section{Synthesis and characterization of conjugates}

The cathepsin B-cleavable peptide, GFLG, was conjugated to the carboxylic acid group of a dendrimer molecule, and MPEG was then attached to the end of peptide (Figure 1). The 12 carboxylic acid moiety of the dendrimer (Figure S1) was then activated with 12 equivalents of EDAC/NHS. The NHS-activated end group of the dendrimer was then conjugated with 13 equivalents of GFLG peptide (Figures S2 and S3). Next, the unreacted GFLG was removed by dialysis, and the lyophilized solid was then used for further conjugation. The degree of conjugation of GFLG to the dendrimer was estimated with a dimethyl group at $0.8 \mathrm{ppm}$ of GFLG peptide and an ethyl proton of the dendrimer at 1.6-2.3 ppm. The degree of substitution of the GFLG peptide was approximately 10.8 for one dendrimer molecule. The carboxylic acid end group of GFLG was then activated again with EDAC and NHS, and conjugated with 4 equivalents of MPEG-amine (Figure S4). Because specific peaks of the ethyl protons of PEG overlapped at 3.5-4.0 ppm with the dendrimer molecule, the conjugation yield of MPEG was estimated by measurement of the weight of the final product. From this measurement, the conjugation yield of MPEG was approximately 3.78 for one dendrimer-GFLG conjugate. Doxorubicin was conjugated to the remaining carboxylic end groups of the GFLG peptide. The doxorubicin content in the DendGDP conjugates was approximately $17.6 \%(\mathrm{w} / \mathrm{w})$ (Table 1).

A DendDP conjugate (Figure S5) was also synthesized for comparison with the DendGDP conjugate. The carboxylic acid group of the dendrimer molecule was activated with EDAC/NHS, and 4 equivalents of MPEG-amine were then attached to the NHS-activated dendrimer (data not shown). The conjugation yield of MPEG was approximately 3.83 for one dendrimer molecule. Doxorubicin was conjugated to the remaining carboxylic end groups of the dendrimer-MPEG conjugate. The doxorubicin content in the DendDP conjugate was approximately $24.8 \%(\mathrm{w} / \mathrm{w})$.

\section{Preparation and characterization of nanoparticles}

The DendDP and DendGDP nanoparticles were prepared using a dialysis procedure. The DendDP and DendGDP nanoparticles had a particle size less than $200 \mathrm{~nm}$ (Figure 2A). 
<smiles>O=C(O)CCC(CCC(=O)O)(CCC(=O)O)NC(=O)CCOCC(COCCC(=O)NC(CCC(=O)O)(CCC(=O)O)CCC(=O)O)(COCCC(=O)NC(CCC(=O)O)(CCC(=O)O)CCC(=O)O)COCCC(=O)NC(CCC(=O)O)(CCC(=O)O)CCC(=O)O</smiles>

$\mathbf{R}=\mathbf{G F L G}$ peptide or $\mathbf{H}$<smiles>CC(C)C[C@H](NC(=O)C(Cc1ccccc1)NC(=O)CN)C(=O)NCC(=O)O</smiles>

$\mathbf{R}=$ GFLG-mPEG or GFLG peptide or $\mathbf{H}$<smiles>[R]C(=O)CCC(CCC(=O)NCC(=O)NC(Cc1ccccc1)C(=O)N[C@@H](CC(C)C)C(=O)NCC(=O)NCCOCCOCCN)(CCC(=O)NCC(C)C)NC(=O)C(C)C</smiles>

\section{$\mathbf{R}_{2}=$ GFLG-DOX or GFLG peptide or $H$} or GFLG-mPEG

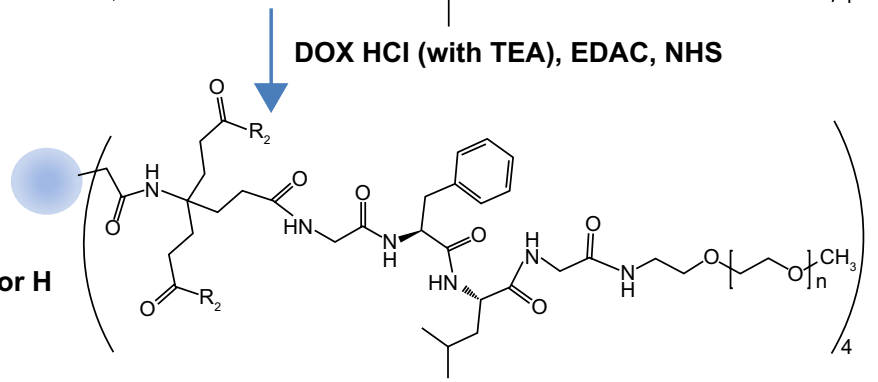

Figure I Synthesis scheme for DendGDP.

Abbreviations: DendGDP, dendrimer-MPEG-DOX conjugate with GFLG peptide linkage; DendDP, dendrimer-MPEG-DOX conjugate; DOX, doxorubicin; MPEG, methoxy poly(ethylene glycol); EDAC, N-(3-dimethylaminopropyl)-N-ethylcarbodiimide hydrochloride; GFLG, Gly-Phe-Leu-Gly; TEA, triethylamine; NHS, N-hydroxysuccinimide.
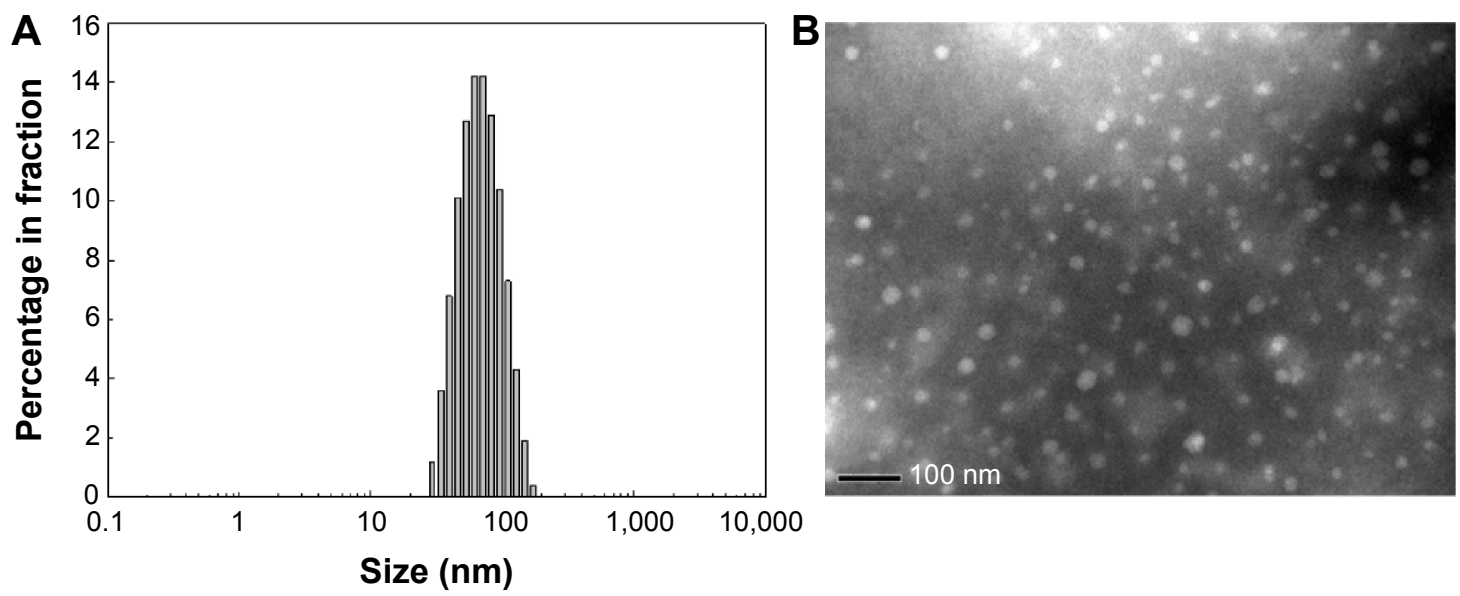

Figure 2 (A) Particle size distribution and (B) transmission electron micrograph of DendDP and DendGDP nanoparticles.

Abbreviations: DendGDP, dendrimer-MPEG-DOX conjugate with GFLG peptide linkage; DendDP, dendrimer-MPEG-DOX conjugate; DOX, doxorubicin; MPEG, methoxy poly(ethylene glycol); GFLG, Gly-Phe-Leu-Gly. 
The DendGDP nanoparticles were slightly bigger than the DendDP nanoparticles. Further, the DendDP and DendGDP nanoparticles had a spherical shape, with a small size in the range of approximately 50-100 nm (Figure 2B). The DendDP and DendGDP nanoparticles also had a core-shell structure, ie, doxorubicin and/or the GFLG peptide-conjugated dendrimer formed the core of each nanoparticle while PEG formed its outer shell (Figure S6). The particle size on transmission electron microscopy was slightly smaller than that obtained by actual measurement of particle size. These differences might be due to the fact that nanoparticles must be shrank by the time of TEM observation while they were hydrated in the aqueous solution.

To study the cathepsin B enzyme-mediated drug release properties of the nanoparticles, DendGDP was incubated at $37^{\circ} \mathrm{C}$ in the presence or absence of cathepsin B, followed by measurement of fluorescence intensity (Figure 3). Since doxorubicin itself has fluorescence properties, the changes
A
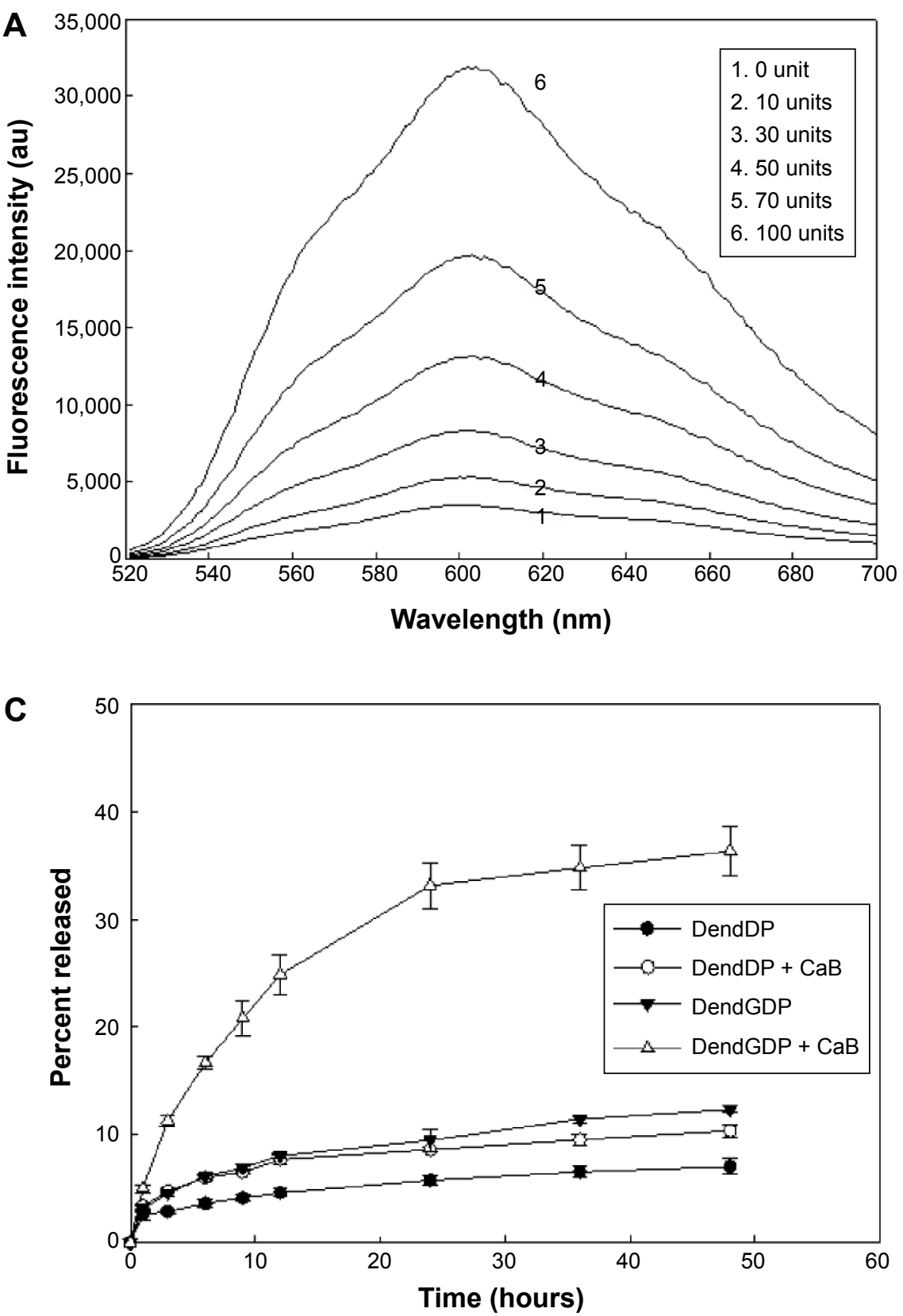

B

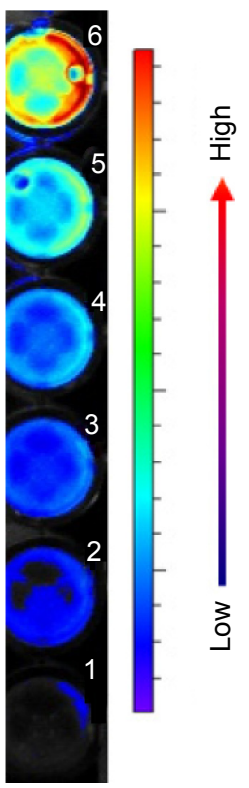

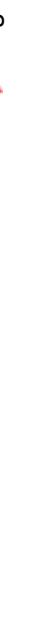


in fluorescence intensity arising from the nanoparticles were measured at 520-700 nm. As shown in Figure 3A and $\mathrm{B}$, the fluorescence intensity increased gradually with increasing cathepsin B activity. Near-infrared fluorescence images (Figure 3B) also indicated an increase in fluorescence intensity. These results suggest that doxorubicin was released via biodegradation of the GFLG peptide upon exposure to cathepsin B, and then fluorescence intensity was increased. Further, the rate of release of doxorubicin was significantly increased in response to treatment with cathepsin B (Figure 3C). Otherwise, DendDP showed a small increase in the rate of release of doxorubicin upon addition of cathepsin $\mathrm{B}$. These results might reflect the fact that the peptide bond formed between doxorubicin and the dendrimer molecule is not specifically degraded by cathepsin B. Otherwise, the rate of release of doxorubicin was significantly increased by degradation of the GFLG peptide in the DendGDP nanoparticles.

\section{In vitro cell culture study}

CT26 colon carcinoma cells were treated with doxorubicin, DendDP, or DendGDP nanoparticles to investigate their anticancer activity. As shown in Figure 4, growth of CT26 cells was dose-dependently inhibited in response to treatment with doxorubicin. CT26 cells treated with DendDP showed a relatively lower potential for growth inhibition (Figure 4A) even though observation of fluorescence intensity indicated higher uptake efficiency by tumor cells when compared with uptake of doxorubicin alone (Figure 4B). A reason for this observation might be the delayed release of doxorubicin from the DendDP nanoparticles (Figure 3), such that cell viability was not significantly changed. As shown in Figure 3, release of doxorubicin from the DendDP nanoparticles was slightly increased when cathepsin B was added, and non-specific release of the drug could have contributed to inhibition of cell growth. Otherwise, growth of CT26 cells in response to treatment with DendGDP was significantly inhibited to a greater extent than with doxorubicin alone or with the DendDP nanoparticles (Figure 4A). Further, the fluorescence intensity in cells treated with DendGDP was also higher than that in cells treated with doxorubicin or DendDP (Figure 4B), indicating that the GFLG peptide underwent enzymatic degradation and doxorubicin was released rapidly from the DendGDP nanoparticles, thereby affecting cell growth. Cathepsin B zymography was performed to confirm cathepsin $B$ activity in CT26 cells, and a specific band was confirmed at $20-35 \mathrm{kDa}$ (data not shown), indicating that these cells expressed cathepsin B activity.

\section{In vivo antitumor activity study}

To study the ability of DendGDP nanoparticles to target tumor tissue, CT26 cells were implanted on the backs of nude mice, and the solid tumors that developed were observed 2 weeks later. Doxorubicin, DendDP nanoparticles, or DendGDP nanoparticles were injected via the tail vein, and the biodistribution of each compound was observed by near-infrared fluorescence imaging. The near-infrared images (Figure 5A) showed that the fluorescence intensity in the tumor xenograft was relatively stronger in response to injection of DendDP or DendGDP nanoparticles than after injection of doxorubicin alone. In particular, injection of doxorubicin resulted in weak fluorescence intensity in the solid tumor tissue while strong fluorescence intensity was observed in other organs. Further, the biodistribution study showed that doxorubicin was predominantly distributed to the kidneys, liver, and brain rather than to the solid tumor tissue. Otherwise, the DendDP and DendGDP nanoparticles showed relatively higher fluorescence intensity at the solid tumor site when compared with the organs. Interestingly, the injected DendGDP nanoparticles showed strong fluorescence intensity at the tumor site as early as 48 hours post injection, while the injected DendDP nanoparticles showed fluorescence intensity at both the solid tumor site and other body sites. Further, in terms of organ distribution, the injected DendGDP nanoparticles showed significantly higher fluorescence intensity at the tumor site than in the organs. Even though the DendDP nanoparticles also showed strong fluorescence intensity at the tumor site, they were also distributed to a considerable extent in the liver, kidneys, and lungs. These results indicate that DendGDP nanoparticles have specificity for CT26 solid tumors and tumor-targeting properties.

\section{Discussion}

Since dendrimers have unique properties, including a hyperbranched structure, they have been extensively investigated as drug-targeting vehicles when conjugated with bioactive agents. ${ }^{19-23}$ Various molecules, including an anticancer drug, a targeting moiety, a peptide, and PEG can be simultaneously attached in the terminal group of a dendrimer molecule. Especially, dendrimer itself having various biomolecules has nanostructural properties in aqueous solution. For example, a MPEG-conjugated dendrimer has a core-shell structure, ie, PEG can form an outer shell on the conjugate and a drug-conjugated dendrimer can form the inner core of the nanoparticle, as shown in Figure S6. Furthermore, a targeting moiety can also be decorated on the corona of the structure. Jain and Jain have reported 

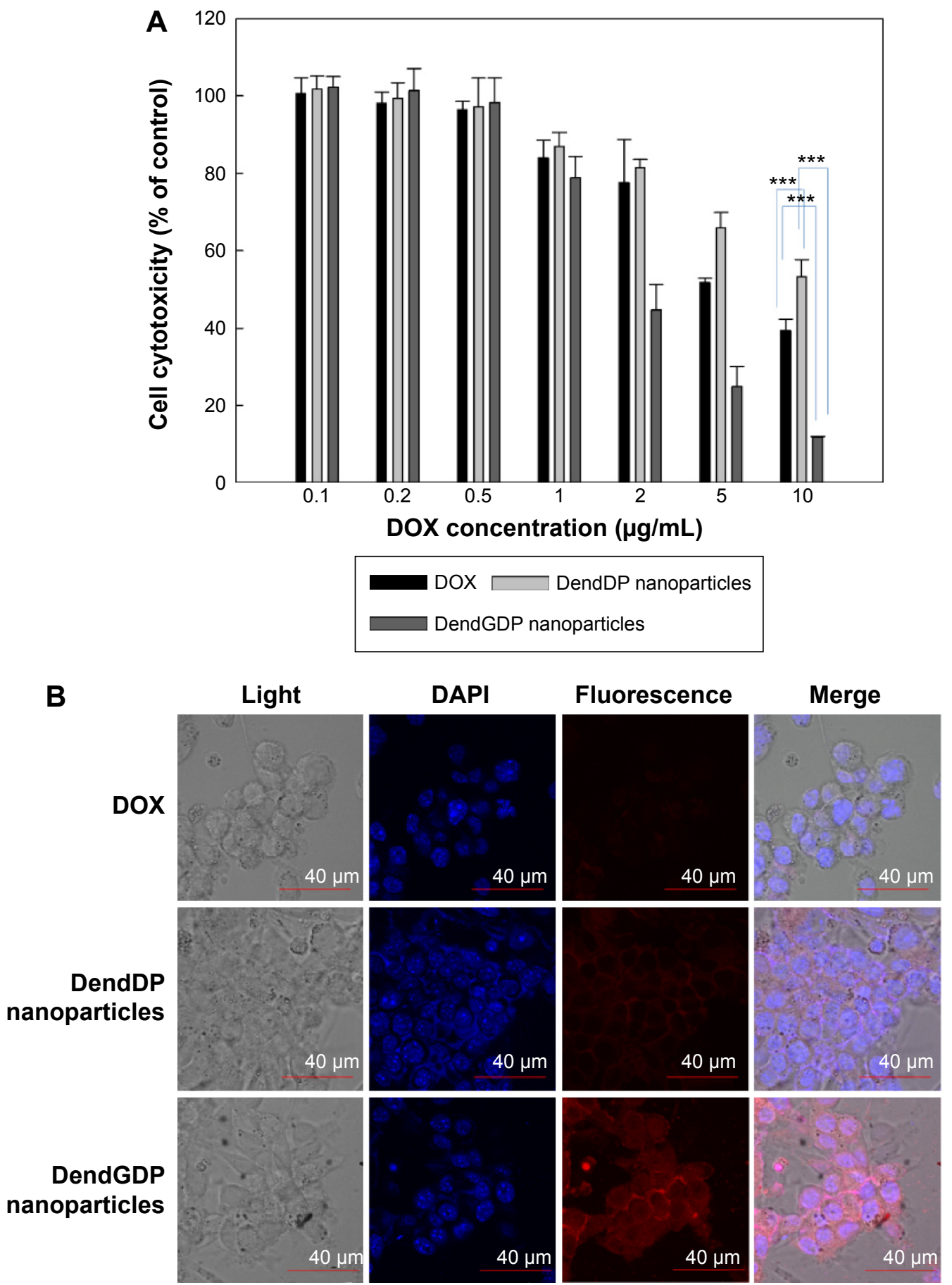

Figure 4 Anticancer activity in CT26 colorectal carcinoma cells.

Notes: (A) Cell growth. (B) Fluorescence intensity in CT26 cells. $* * * P<0.00$ I

Abbreviations: DendGDP, dendrimer-MPEG-DOX conjugate with GFLG peptide linkage; DendDP, dendrimer-MPEG-DOX conjugate; DOX, doxorubicin; MPEG, methoxy poly(ethylene glycol); GFLG, Gly-Phe-Leu-Gly; DAPI, 4,6-diamidino-2-phenylindole.

on an arginine-conjugated poly(propylene) imine that was conjugated with endostatin and doxorubicin for dual attack on cancer, ie, inhibition of angiogenesis can be achieved by endostatin release and killing of cancer cells can be achieved by doxorubicin. ${ }^{20}$ Further, Yang et al synthesized PAMAM conjugated with alkali blue for targeting of the lymphatics. ${ }^{23}$
They showed that this alkali blue-conjugated PAMAM dendrimer enhanced lymphatic drainage and had a longer residence time in the lymph nodes when compared with methylene blue solution. He et al reported that a RGD peptideconjugated PAMAM dendrimer can be used as a targeting vehicle for cancer cells overexpressing $\alpha_{\mathrm{v}} \beta 3$ integrin and that 

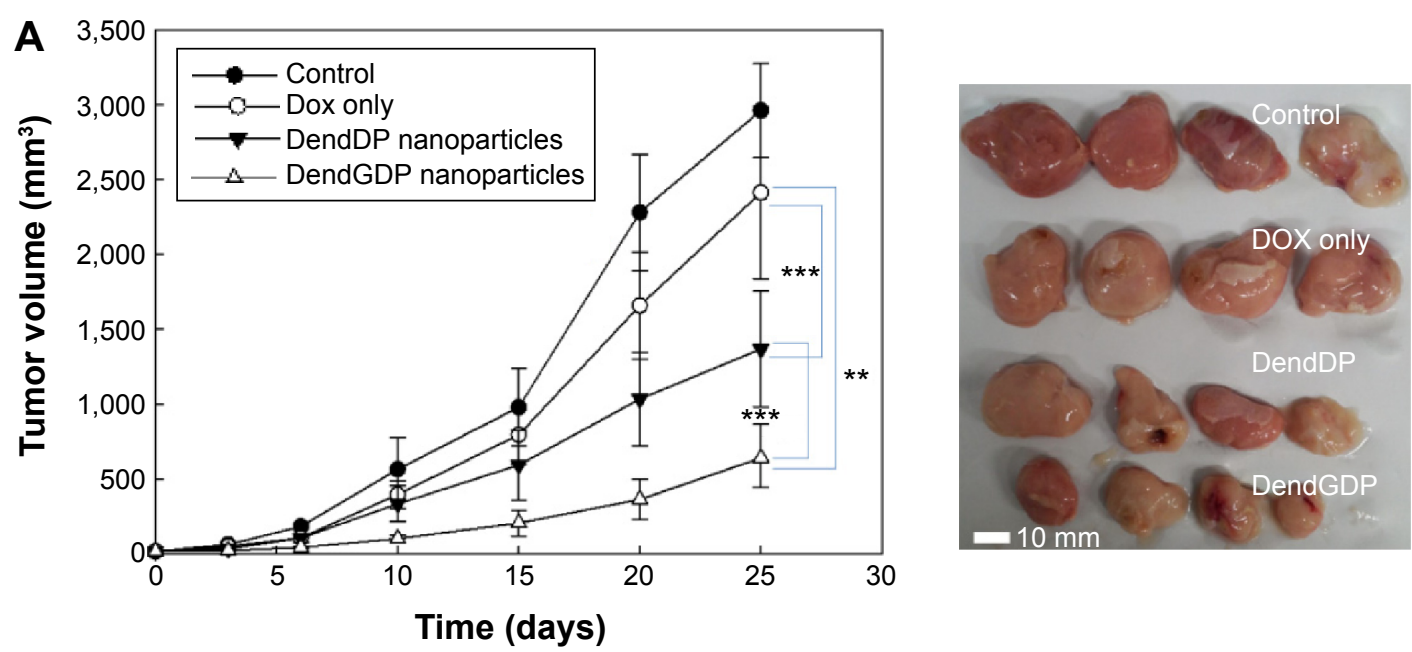

\section{B 24 hours}
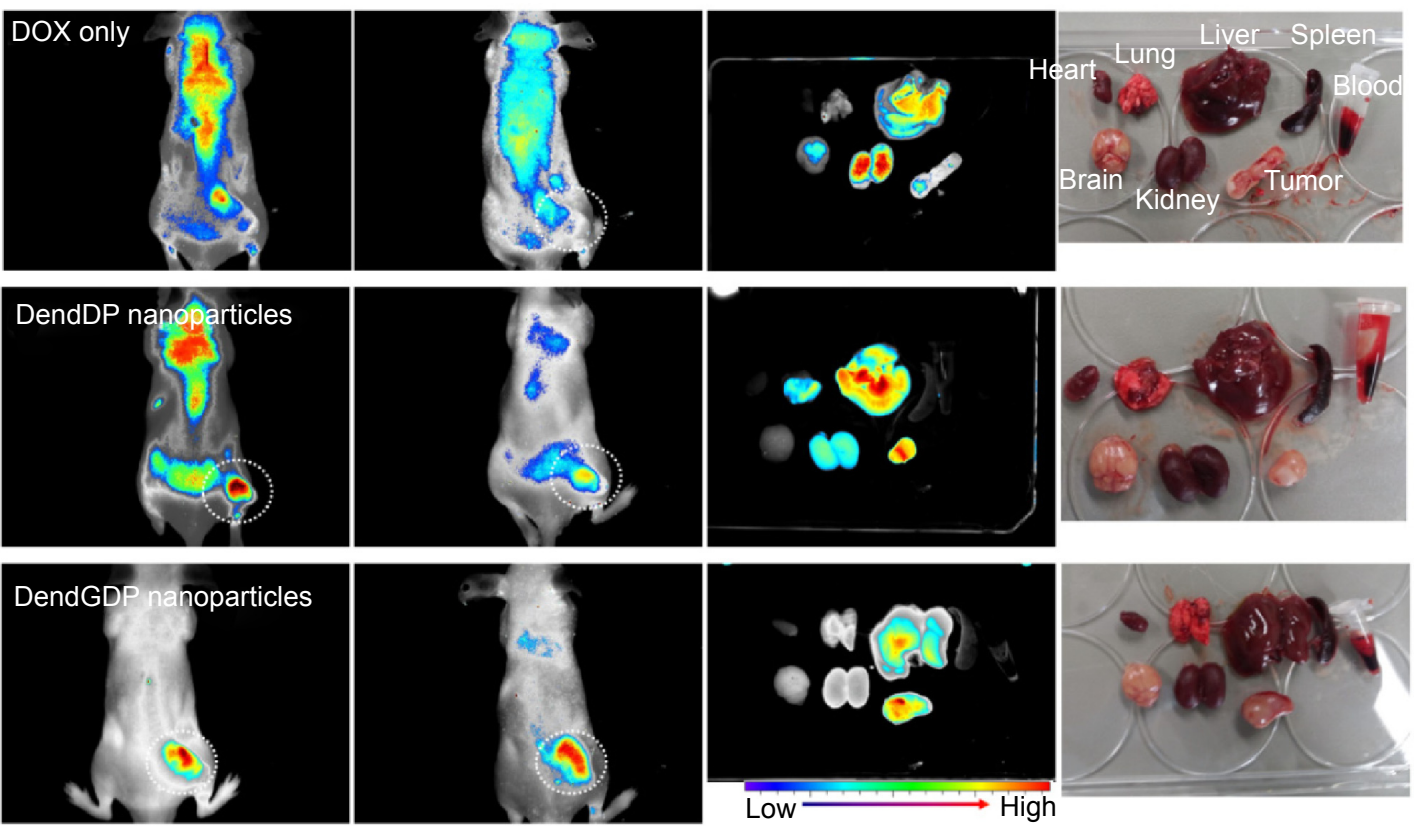

Figure 5 Anticancer activity of dendrimer nanoparticles.

Notes: (A) Anticancer activity of dendrimer nanoparticles and (B) near-infrared fluorescence images for CT26-bearing male nude BALB/c mice. DOX itself or DOXconjugated dendrimer nanoparticles $(5 \mathrm{mg} / \mathrm{kg}$ ) were injected into the tail vein of each mouse. The major organs were removed from each mouse 48 hours after injection (Dotted circle: solid tumor). The data shown as the mean \pm standard deviation $(n=4)$. $* * * P<0.01 ; * * P<0.001$

Abbreviations: DendGDP, dendrimer-MPEG-DOX conjugate with GFLG peptide linkage; DendDP, dendrimer-MPEG-DOX conjugate; DOX, doxorubicin; MPEG, methoxy poly(ethylene glycol); GFLG, Gly-Phe-Leu-Gly.

doxorubicin was entrapped in the hydrophobic dendritic core of the aggregates. ${ }^{24}$ These authors confirmed that this RGD peptide-conjugated dendrimer targeted cancer cells through specific interaction with $\alpha_{v} \beta 3$ integrin.

We synthesized a PEG and DOX-conjugated dendrimer using a GFLG spacer to target cancer cells via cathepsin B-mediated drug release. Since cathepsin B is specifically excreted by cancer cells during invasion or metastasis, a cathepsin B-sensitive peptide, ie, GFLG, can be used for cancer cell targeting through conjugation of anticancer drugs. ${ }^{26}$ DendGDP nanoparticles showed cathepsin B-mediated drug release and tumor targeting, while DendDP, a non-cleavable conjugate, was not sensitive with cathepsin B (Figures 3 and 5). As shown in Figure 5, treatment with DendGDP showed strong fluorescence intensity at tumor sites in mice, with minimal fluorescence intensity at other body sites. However, fluorescence intensity of DenDP treatment group was relatively stronger at liver tissues than tumor tissues (Figure 5C). Duncan et al reported that DOX-N-(2hydroxypropyl)-methacrylamide conjugates using cathepsin 
B-cleavable peptide linkage showed approximately 6-fold accumulation in tumor tissue when compared with free doxorubicin. ${ }^{27}$ Chen et al also reported that a cell-penetrating doxorubicin-peptide conjugate using GFLG showed cathepsin B-mediated drug release properties and an ability to target cancer cells. ${ }^{28}$ Furthermore, sorbitol linked with a cathepsin B-cleavable peptide and doxorubicin showed cathepsin B-mediated drug release kinetics and cancer cell specificity. ${ }^{29}$ Poly(L-lysine)-PEG copolymers with near-infrared fluorescence probes can be used to detect orthotopically implanted colon cancer model of CT2 6 cells. ${ }^{30}$ Our results also showed that DendGDP became relatively concentrated in CT26 solid tumors when compared with DendDP or doxorubicin alone (Figure 5C). DendGDP has the highest fluorescence intensity in the tumor tissue rather than in the body organs, while doxorubicin alone and DendDP showed strong fluorescence in the organs, indicating that DendGDP has potential for use as a diagnostic tool for cancer. Adenis et al reported that expression of cathepsin B was significantly elevated in malignant colorectal carcinoma when compared with normal adjacent colorectal tissue. ${ }^{31}$ They also suggested that cathepsin B expression can be used to monitor progression of colorectal cancer. Members of the cathepsin family are known to have a strong relationship with invasion and metastasis of colorectal cancer and are used for prognostication. ${ }^{32}$ Furthermore, Funovics et al showed that levels of cathepsin B gradually increased from healthy colon to adenoma to adenocarcinoma in a CT26 cell-implanted mouse model of colorectal carcinoma. ${ }^{33}$

In conclusion, we synthesized a dendrimer-DOX conjugate via a cathepsin B-cleavable peptide. This nanoconjugate had a small particle size. DendGDP had cathepsin B-sensitive drug release properties, whereas the drug release kinetics of DendDP were not significantly changed. Further, DendGDP had improved anticancer activity when compared with doxorubicin or DendDP in an vivo mouse CT26 tumor xenograft model. DendGDP became relatively concentrated in the tumor tissue and had stronger fluorescence intensity compared with doxorubicin alone or DendDP nanoparticles. We suggest that DendGDP can be used as a promising vehicle for cancer cell targeting.

\section{Acknowledgment}

This study was supported by a grant (2015-25) from the Biomedical Research Institute, Pusan National University Hospital.

\section{Disclosure}

The authors report no conflicts of interest in this work.

\section{References}

1. Mitragotri S, Burke PA, Langer R. Overcoming the challenges in administering biopharmaceuticals: formulation and delivery strategies. Nat Rev Drug Discov. 2014;13:655-672.

2. McLornan DP, List A, Mufti GJ. Applying synthetic lethality for the selective targeting of cancer. $N$ Engl J Med. 2014;371:1725-1735.

3. Rousseau A, Marquet P. Application of pharmacokinetic modelling to the routine therapeutic drug monitoring of anticancer drugs. Fundam Clin Pharmacol. 2002;16:253-262.

4. Paci A, Veal G, Bardin C, et al. Review of therapeutic drug monitoring of anticancer drugs. Part 1. Cytotoxics. Eur J Cancer. 2014;50: 2010-2019.

5. Widmer N, Bardin C, Chatelut E, et al. Review of therapeutic drug monitoring of anticancer drugs. Part two - targeted therapies. Eur J Cancer. 2014;50:2020-2036.

6. Marchetti C, Palaia I, Giorgini M, et al. Targeted drug delivery via folate receptors in recurrent ovarian cancer: a review. Onco Targets Ther. 2014; 7:1223-1236.

7. McCrudden CM, McCarthy HO. Current status of gene therapy for breast cancer: progress and challenges. Appl Clin Genet. 2014;7:209-220.

8. Ryan SM, Brayden DJ. Progress in the delivery of nanoparticle constructs: towards clinical translation. Curr Opin Pharmacol. 2014; $18 \mathrm{C}: 120-128$

9. Kajimoto K, Sato Y, Nakamura T, Yamada Y, Harashima H. Multifunctional envelope-type nano device for controlled intracellular trafficking and selective targeting in vivo. J Control Release. 2014;190: 593-606.

10. Nichols JW, Bae YH. EPR: evidence and fallacy.J Control Release. 2014; 190:451-464.

11. Cho HY, Lee YB. Nano-sized drug delivery systems for lymphatic delivery. J Nanosci Nanotechnol. 2014;14:868-880.

12. Orza A, Casciano D, Biris A. Nanomaterials for targeted drug delivery to cancer stem cells. Drug Metab Rev. 2014;46:191-206.

13. Hubbell JA, Chikoti A. Nanomaterials for drug delivery. Science. 2012; 337:303-305.

14. Kapse-Mistry S, Govender T, Srivastava R, Yergeri M. Nanodrug delivery in reversing multidrug resistance in cancer cells. Front Pharmacol. 2014;5:159

15. Beg S, Rizwan M, Sheikh AM, Hasnain MS, Anwer K, Kohli K. Advancement in carbon nanotubes: basics, biomedical applications and toxicity. J Pharm Pharmacol. 2011;63:141-163.

16. Cabral H, Kataoka K. Progress of drug-loaded polymeric micelles into clinical studies. J Control Release. 2014;190:465-476.

17. Zhao Y, Lin D, Wu F, et al. Discovery and in vivo evaluation of novel RGD-modified lipid-polymer hybrid nanoparticles for targeted drug delivery. Int J Mol Sci. 2014;15:17565-17576.

18. Jeong YI, Kim YW, Jung S, et al. Delivery of transferrin-conjugated polysaccharide nanoparticles in 9L gliosarcoma cells. $J$ Nanosci Nanotechnol. 2015;15:125-129.

19. Abbasi E, Aval SF, Akbarzadeh A, et al. Dendrimers: synthesis, applications, and properties. Nanoscale Res Lett. 2014;9:247.

20. Jain K, Jain NK. Surface engineered dendrimers as antiangiogenic agent and carrier for anticancer drug: dual attack on cancer. J Nanosci Nanotechnol. 2014;14:5075-5087.

21. Lyulin SV, Vattulainen I, Gurtovenko AA. Complexes comprised of charged dendrimers, linear polyelectrolytes, and counterions: insight through coarse-grained molecular dynamics simulations. Macromolecules. 2008;41:4961-4968.

22. Eleftheriou E, Karatasos K. Modeling the formation of ordered nanoassemblies comprised by dendrimers and linear polyelectrolytes: the role of Coulombic interactions. J Chem Phys. 2012;137:144905.

23. Yang R, Xia S, Ye T, et al. Synthesis of a novel polyamidoamine dendrimer conjugating with alkali blue as a lymphatic tracer and study on the lymphatic targeting in vivo. Drug Deliv. November 19, 2014. [Epub ahead of print]

24. He X, Alves CS, Oliveira N, et al. RGD peptide-modified multifunctional dendrimer platform for drug encapsulation and targeted inhibition of cancer cells. Colloids Surf B Biointerfaces. 2015;125:82-89. 
25. Kaminskas LM, Kelly BD, McLeod VM, et al. Capping methotrexate $\alpha$-carboxyl groups enhances systemic exposure and retains the cytotoxicity of drug conjugated PEGylated polylysine dendrimers. $\mathrm{Mol}$ Pharm. 2011;8:338-349.

26. Bervar A, Zajc I, Sever N, Katunuma N, Sloane BF, Lah TT. Invasiveness of transformed human breast epithelial cell lines is related to cathepsin B and inhibited by cysteine proteinase inhibitors. Biol Chem. 2003;384:447-455.

27. Duncan R, Sat-Klopsch YN, Burger AM, Bibby MC, Fiebig HH, Sausville EA. Validation of tumour models for use in anticancer nanomedicine evaluation: the EPR effect and cathepsin B-mediated drug release rate. Cancer Chemother Pharmacol. 2013;72:417-427.

28. Chen Z, Zhang P, Cheetham AG, et al. Controlled release of free doxorubicin from peptide-drug conjugates by drug loading. J Control Release. 2014;191:123-130.

29. Maniganda S, Sankar V, Nair JB, Raghu KG, Maiti KK. A lysosometargeted drug delivery system based on sorbitol backbone towards efficient cancer therapy. Org Biomol Chem. 2014;12:6564-6569.
30. Alencar H, Funovics MA, Figueiredo J, Sawaya H, Weissleder R, Mahmood U. Colonic adenocarcinomas: near-infrared microcatheter imaging of smart probes for early detection - study in mice. Radiology. 2007; 244:232-238.

31. Adenis A, Huet G, Zerimech F, Hecquet B, Balduyck M, Peyrat JP. Cathepsin B, L, and D activities in colorectal carcinomas: relationship with clinico-pathological parameters. Cancer Lett. 1995;96:267-275.

32. Kuester D, Lippert H, Roessner A, Krueger S. The cathepsin family and their role in colorectal cancer. Pathol Res Pract. 2008;204:491-500.

33. Funovics MA, Alencar H, Montet X, Weissleder R, Mahmood U. Simultaneous fluorescence imaging of protease expression and vascularity during murine colonoscopy for colonic lesion characterization. Gastrointest Endosc. 2006;64:589-597. 


\section{Supplementary materials}

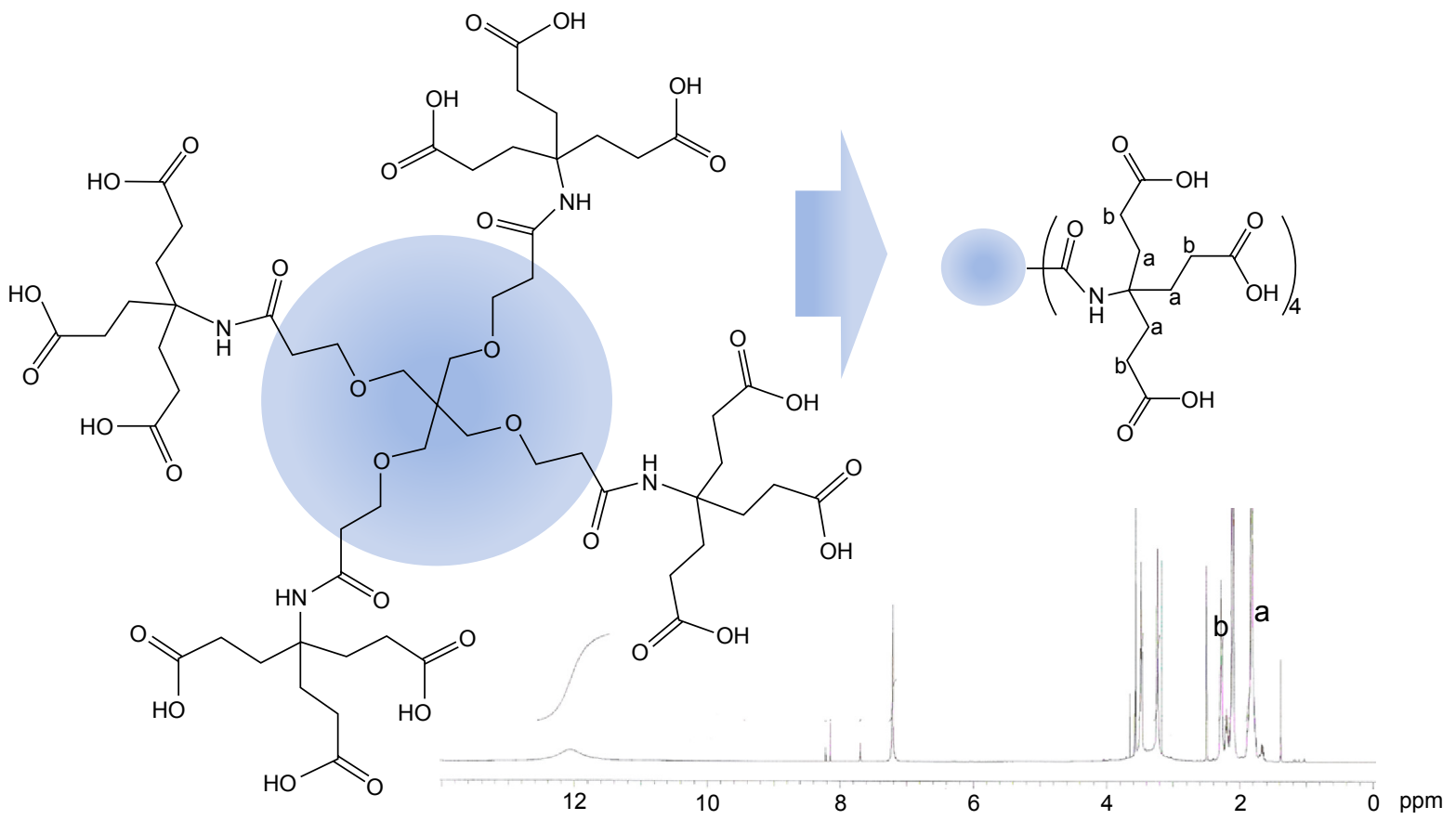

Figure SI 'H nuclear magnetic resonance spectra for the dendrimer molecule. Note: Peaks of a and $b$ was indicated methylene protons of dendrimer core.

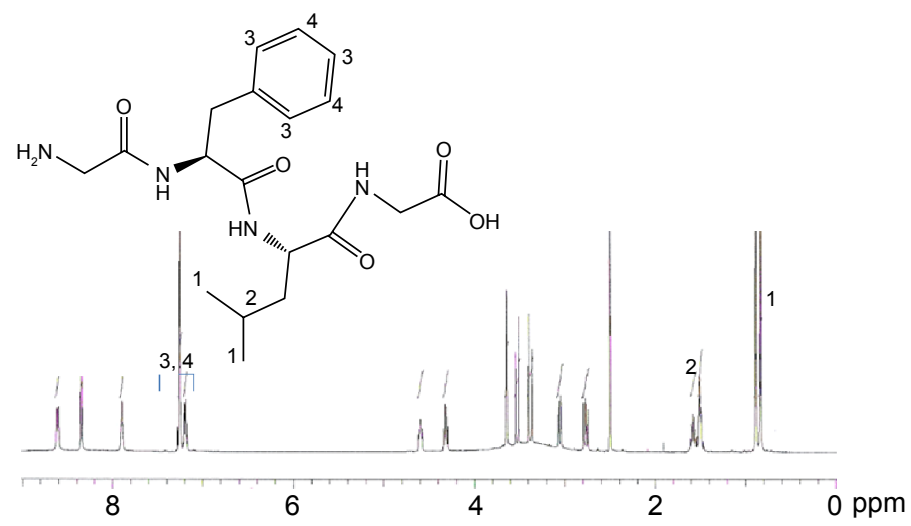

Figure S2 'H nuclear magnetic resonance spectra for the Gly-Phe-Leu-Gly peptide. 


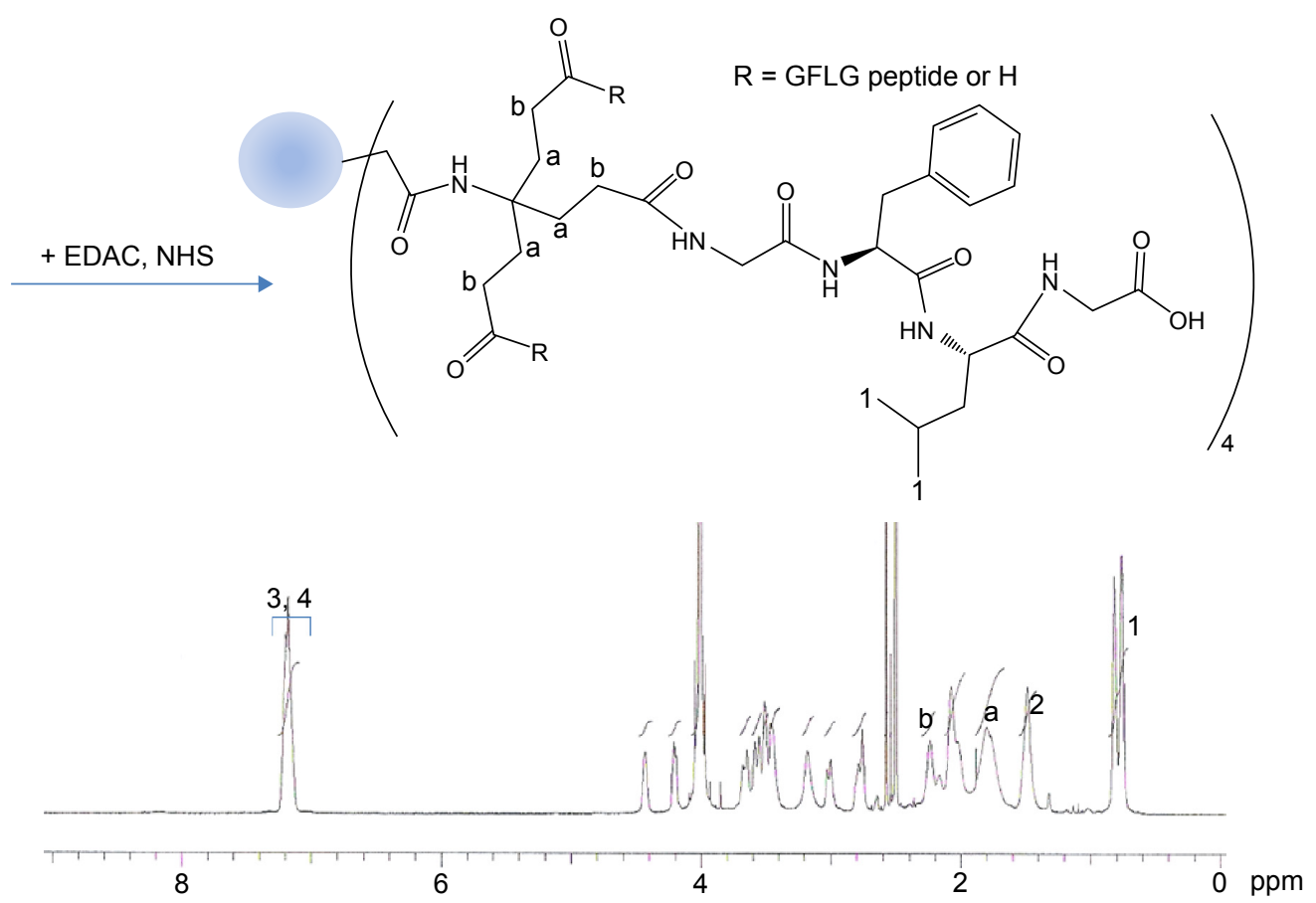

Figure S3 'H nuclear magnetic resonance spectra for dendrimer-GFLG conjugate.

Note: Peaks of a and $b$ was indicated methylene protons of dendrimer core.

Abbreviations: GFLG, Gly-Phe-Leu-Gly; EDAC, N-(3-dimethylaminopropyl)-N-ethylcarbodiimide hydrochloride; NHS, N-hydroxysuccinimide.

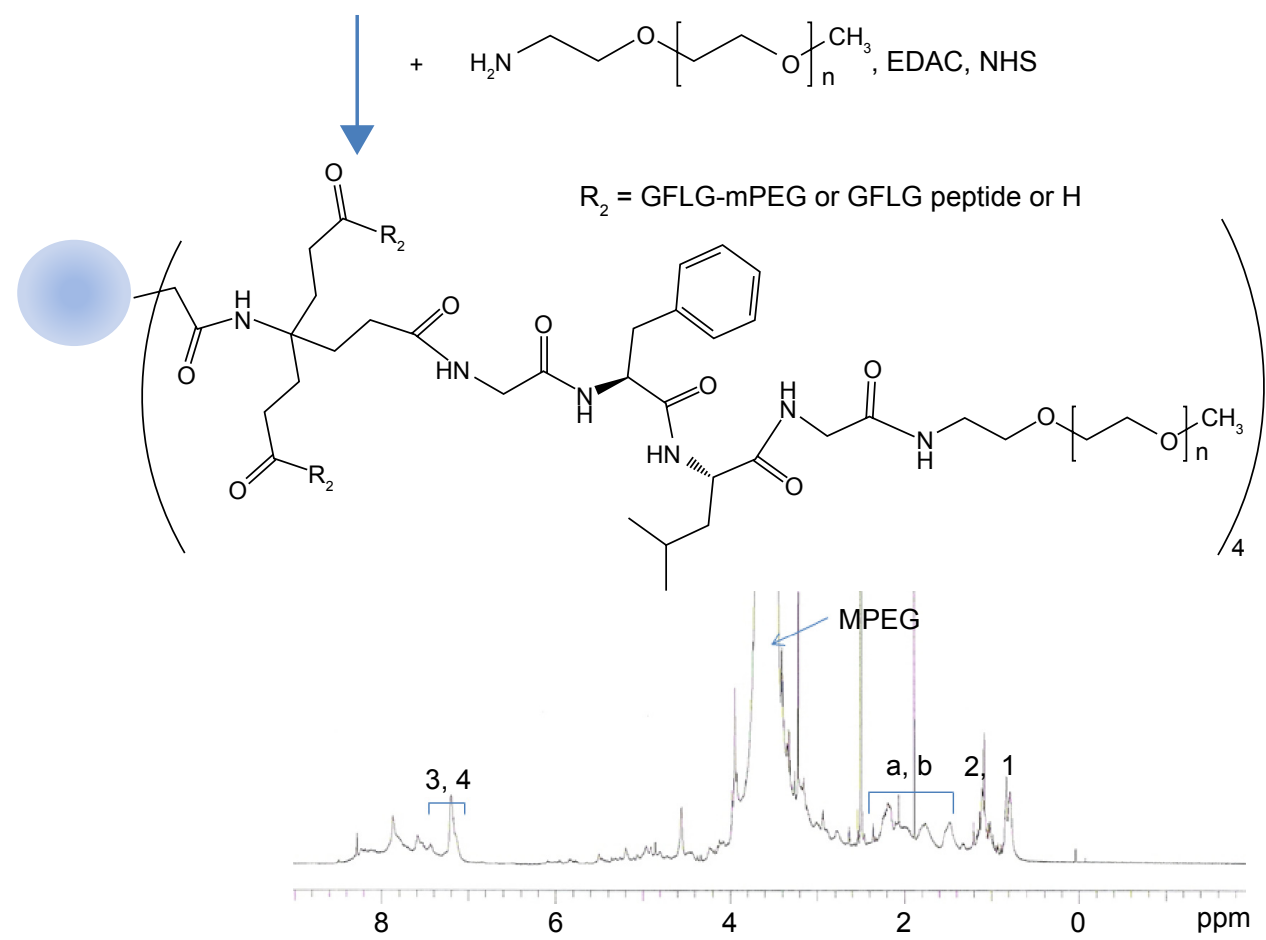

Figure S4 'H nuclear magnetic resonance spectra for the dendrimer-GFLG-mPEG conjugate.

Note: Peaks of $a$ and $b$ was indicated methylene protons of dendrimer core.

Abbreviations: GFLG, Gly-Phe-Leu-Gly; EDAC, N-(3-dimethylaminopropyl)-N-ethylcarbodiimide hydrochloride; NHS, N-hydroxysuccinimide; PEG, poly(ethylene glycol); MPEG, methoxy poly(ethylene glycol). 


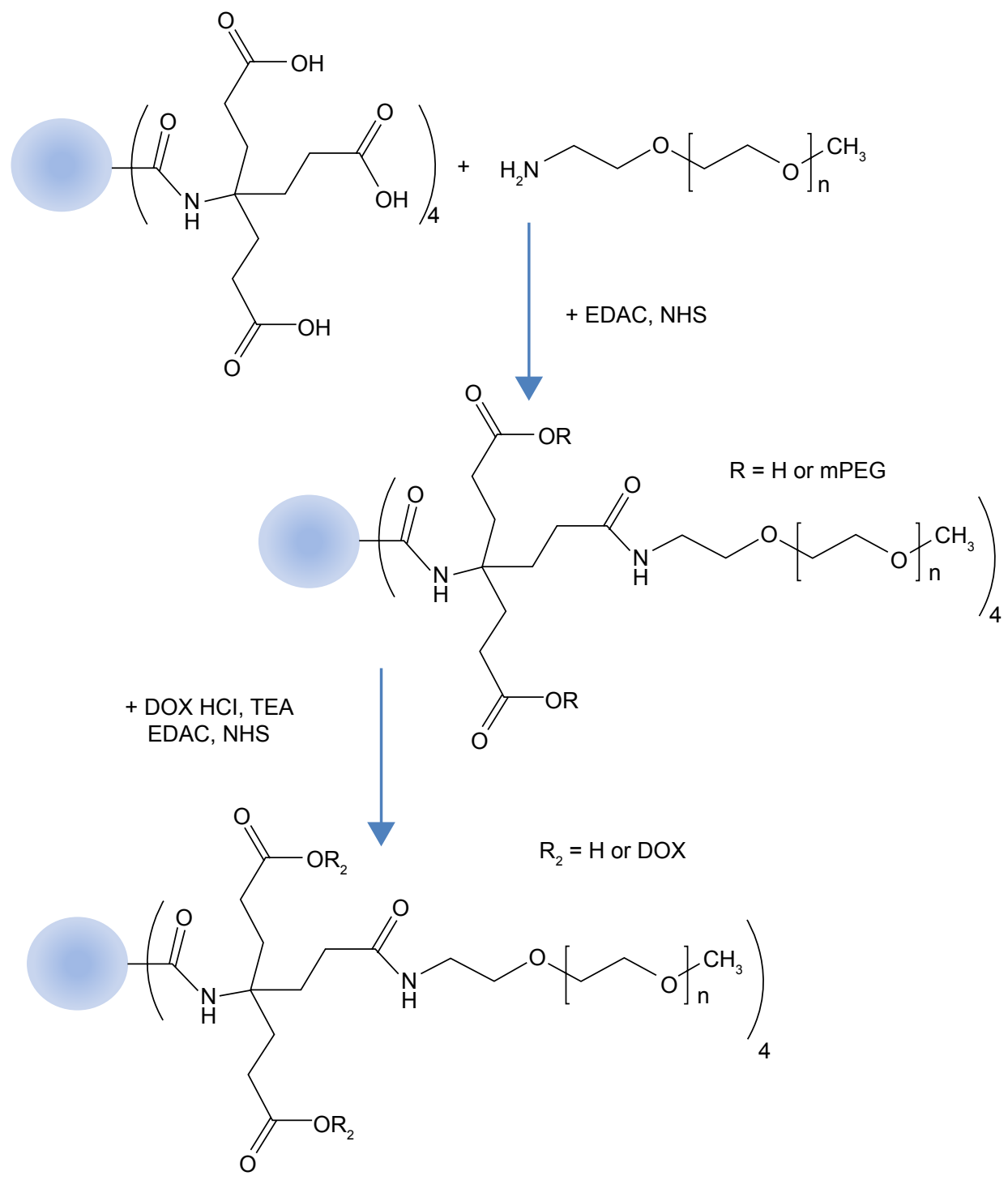

Figure S5 Synthesis scheme for DendDP conjugate.

Abbreviations: DendDP, dendrimer-MPEG-DOX conjugate; EDAC, N-(3-dimethylaminopropyl)-N-ethylcarbodiimide hydrochloride; DOX, doxorubicin; NHS, N-hydroxysuccinimide; MPEG, methoxy poly(ethylene glycol); TEA, triethylamine.

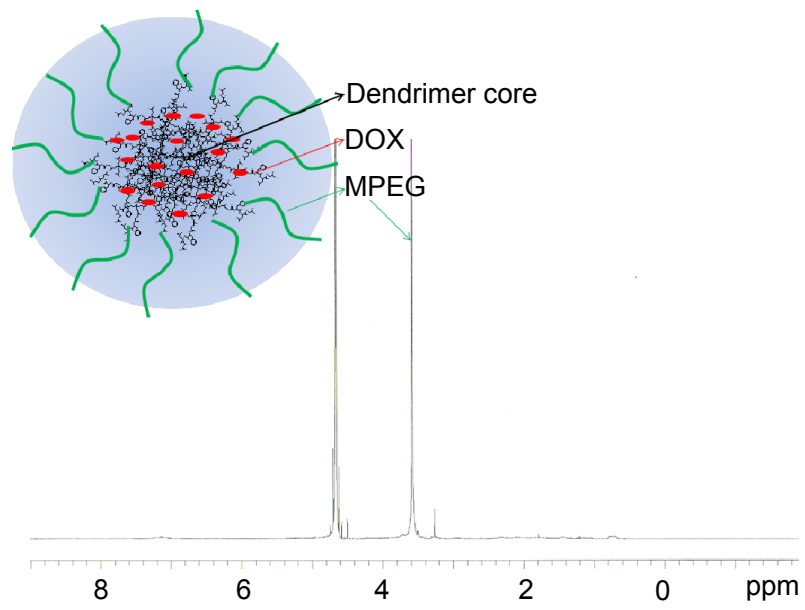

Figure S6 ' $\mathrm{H}$ nuclear magnetic resonance spectra for DendGDP nanoparticles in double-distilled water.

Abbreviations: DendGDP, dendrimer-MPEG-DOX conjugate with GFLG peptide linkage; DOX, doxorubicin; MPEG, methoxy poly(ethylene glycol); GFLG, Gly-Phe-Leu-Gly; Ppm, part per million. 
International Journal of Nanomedicine

Dovepress

\section{Publish your work in this journal}

The International Journal of Nanomedicine is an international, peerreviewed journal focusing on the application of nanotechnology in diagnostics, therapeutics, and drug delivery systems throughou the biomedical field. This journal is indexed on PubMed Central, MedLine, CAS, SciSearch ${ }^{\circledR}$, Current Contents ${ }^{\circledR} /$ Clinical Medicine,
Journal Citation Reports/Science Edition, EMBase, Scopus and the Elsevier Bibliographic databases. The manuscript management system is completely online and includes a very quick and fair peer-review system, which is all easy to use. Visit http://www.dovepress.com/ testimonials.php to read real quotes from published authors.

Submit your manuscript here: http://www.dovepress.com/international-journal-of-nanomedicine-journal 